\title{
Deux protéines de fusion dans la leucémie aiguë promyélocytaire
}

La translocation $(15 ; 17)$ associéc aux leucémies aiguës promyélocytaircs est à l'origine de la formation d'une protéine hybride entre le gène $m y l$ ou $P M L$ et le récepteur $\alpha$ de l'acide rétinoïque (RAR). A la précédente caractérisation, par trois équipes différentes, du gène $P M L$ et du transcrit hybride, déjà rapportée dans ces colonnes $\left(\mathrm{m} / \mathrm{s} n^{\circ} 8\right.$, vol. 7, p. 880-881) viennent s'ajouter celle d'un groupc de Houston (TX, USA) [1] et celle récemment publiée par l'équipe de P. Chambon (Strasbourg, France) [2], riches d'enseignements nouvcaux.

PML est une protéine nucléaire dont la partie amino-terminale comprend trois groupements riches en cystéines et histidines, rappelant l'agencement de doigts de zinc. Des motifs très analogucs ont été décrits dans une famille de protéines fusionnécs à des protooncogènes et dont le produit chimérique possède des propriétés transformantes (T18 [3], RFP [4]). le marquage immunocytochimique de cette protéine donne une répartition nucléaire mouchetéc qui devient homogène après mutation de deux résidus situés dans cette région riche en cystéines.

Dans les ccllules tumorales, la protéine chimérique PML-RAR est présente en quantité supérieure à celle du produit du gène $R A R$ issu de l'allèle non transloqué. Dans différents types de cellules transfectées, cettc protéine hybride est majoritairement cytoplasmique en l'absence d'acide rétinoïque (RA). En revanche, l'addition de RA entraîne sa translocation dans le noyau.

In vitro, la protéine PML-RAR est capable d'hétérodimérisation avec le produit PML. C'est sans doute cette liaison qui est responsable de la colocalisation de ces deux protéines in vivo. Deux principaux types de transcrits chimériques PML-RAR ont été dépistés chez les patients présentant une leucémie aiguë promyélocytaire, diffé- rant uniquement par leur point de cassure dans le gène $P M L$ : le transcrit de type A comprend les 552 premiers résidus de la protéine PML, alors que le transcrit de type B n'en contient que 394 (cette séquence conserve le domaine riche en cystéines et la région en hélice $\alpha$ ). La localisation du point de cassure du gène $R A R$ est, en revanche, située dans le deuxième intron quel que soit le transcrit. Chacun des patients étudiés ne présentc qu'un seul des deux types de transcrits, prouvant ainsi l'origine clonale du processus tumoral. Les transcrits réciproques RAR-PML sont également dépistés et on ne pcut pas exclure lcur intervention dans le blocage de la différenciation promyélocytaire.

Enfin, des expériences de cotransfection de différentes constructions dans deux modèles cellulaires donnent des résultats en accord avec ceux précédemment développés par l'équipe de A. Dejean. En effet, en l'absence de RA, le vecteur d'expression PMLRAR, contrairement au vecteur d'expression RAR $\alpha$, a un effet répresseur sur certains des promoteurs étudiés.

Il semble, d'après ces données, que la protéine PML-RAR intervienne en tant qu'inhibiteur transdominant sur les produits du gène $R A R \alpha$ normal, ou du gène $P M L$ normal ou sur les deux, empêchant leur rôle dans la différenciation ccllulaire. L'augmentation des concentrations de RA permettrait de surpasser cet effet inhibiteur et expliquerait l'effet thérapeutique de l'acide rétinoïque observé chez les patients. $\mathrm{La}$ prochaine étape est désormais de comprendre la part physiologique jouée par la protéine PML d'unc part, et le réceptcur $\alpha$ de l'acide rétinoïque d'autre part, dans la différenciation des promyélocytes en granulocytes.
1. Chang KS, Stass SA, Chu DT, Deaven ILL, Trujillo JM, Friercich EJ. Characterization of a fusion cDNA (RAR/myl) transcribed from the $t(15 ; 17)$ translocation breakpoint in acute promyelocytic leukemia. Mol Cell Biol $1992 ; 12$ : 800-10.

2. Kastner P, Perez A, Lutz Y, et al. Structure, localization and transcriptional properties of two classes of retinoic acid receptor $\alpha$ fusion proteins in acute promyelocytic leukemia (APL) : structural similarities with a new family of oncoproteins. EMBO J $1992 ; 11: 629-42$.

3. Miki $\Gamma$, Fleming TP, Cresscenzi $M$, el al. Development of a highly efficient expression cDNA cloning system. Application to oncogene isolation. Proc Nall Acad Sci USA 1991; 88 5167-71.

4. Takahashi $M$, Inaguma $Y$, Hiai $H$, Hirose F. I Developmentally regulated expression of a human "finger "-containing gene encoded by the 5' half of the ret transforming gene. Mol Cell Biol $1988 ; 8: 1853-6$

H. G. 